\title{
Genetic variants at the 9p21 locus contribute to atherosclerosis through modulation of ANRIL and CDKN2A/B
}

\author{
Ada Congrains a , Kei Kamide a , Ryousuke Oguro ${ }^{a}$, Osamu Yasudab ${ }^{b}$, Keishi Miyatac,d, \\ Eiichiro Yamamoto ${ }^{e}$, Tatsuo Kawai ${ }^{a}$, Hiroshi Kusunoki ${ }^{f}$, Hiroko Yamamoto ${ }^{\text {, }}$, Yasushi Takeya ${ }^{a}$, \\ Koichi Yamamoto ${ }^{a}$, Miyuki Onishi ${ }^{a}$, Ken Sugimoto ${ }^{a}$, Tomohiro Katsuya ${ }^{\mathrm{f}}$, Nobuhisa Awata ${ }^{\mathrm{g}}$, \\ Kazunori Ikebe $^{\mathrm{h}}$, Yasuyuki Gondo ${ }^{\mathrm{i}}$, Yuichi Oike ${ }^{\mathrm{c}}$, Mitsuru Ohishi ${ }^{\mathrm{a}, *}$, Hiromi Rakugi ${ }^{\mathrm{a}}$ \\ a Department of Geriatric Medicine and Nephrology, Osaka University Graduate School of Medicine, Japan \\ ${ }^{b}$ Department of Cardiovascular Clinical and Translational Research, Kumamoto University Hospital, Japan \\ ${ }^{c}$ Department of Molecular Genetics, Graduate School of Medical Sciences, Kumamoto University, Japan \\ d Immunology, Allergy and Vascular Medicine, Graduate School of Medical Sciences, Kumamoto University, Japan \\ e Cardiovascular Medicine, Graduate School of Medical Sciences, Kumamoto University, Japan \\ ${ }^{\mathrm{f}}$ Clinical Gene Therapy, Osaka University Graduate School of Medicine, Japan \\ ${ }^{g}$ Department of Cardiology, Osaka Medical Center for Cancer and Cardiovascular Diseases, Japan \\ h Department of Prosthodontics and Oral Rehabilitation, Osaka University Graduate School of Dentistry, Japan \\ i Department of Clinical Thanatology and Geriatric Behavioral Science, Osaka University, Graduate School of Human Sciences, Japan
}

\section{A R T I C L E I N F O}

\section{Article history:}

Received 13 September 2011

Received in revised form 31 October 2011

Accepted 11 November 2011

Available online 19 November 2011

\section{Keywords:}

Chromosome 9p21

ANRIL

CDKN2A

CDKN2B

Atherosclerosis

\begin{abstract}
A B S T R A C T
Genome-wide association studies (GWAS) have identified genetic variants contributing to the risk of cardiovascular disease (CVD) at the chromosome 9p21 locus. The CVD-associated region is adjacent to the two cyclin dependent kinase inhibitors $(C D K N) 2 A$ and $2 B$ and the last exons of the non-coding RNA, ANRIL. It is still not clear which of or how these transcripts are involved in the pathogenesis of atherosclerosis. Objective: We assessed the hypothesis that 9p21 locus polymorphisms influence the expression of the transcripts in the region (ANRIL, $C D K N 2 A / B$ ) and that these transcripts contribute to atherogenesis through the modulation of proliferation in VSMC.

Methods: We genotyped 18 SNPs $\left(r^{2}<0.8\right.$ and MAF $\left.>0.05\right)$ across the region of interest: $C D K N 2 A / B$ and ANRIL, encompassing the CVD-associated region. RNA and DNA were extracted from the blood of 57 volunteers (69-72 years old). Carotid ultrasound was performed in 56 subjects. CDKN2A/B and ANRIL (exons 1-2 and 17-18) expression was measured employing RT-PCR. Gene expression and cell growth were evaluated in cultured VSMC after the siRNA-mediated knock-down of ANRIL.

Results: The risk alleles for atherosclerosis-related phenotypes were consistently associated with a lower expression of ANRIL when evaluating exons 1-2. Common carotid artery stenosis was associated with a significantly lower $(P<0.01)$ expression of ANRIL (exons $1-2)$. ANRIL knock-down in VSMC caused significant variation in expression of $C D K N 2 A / B(P<0.05)$ and reduction of cell growth $(P<0.05)$ in vitro. Conclusion: Disease-associated SNPs at the 9p21 locus predominantly affect the expression of ANRIL. Overall, our results suggest that several CVD-associated SNPs in the 9p21 locus affect the expression of ANRIL, which, in turn modulate cell growth, possibly via $C D K N 2 A / B$ regulation.
\end{abstract}

(c) 2011 Elsevier Ireland Ltd. All rights reserved.
Genome-wide association studies (GWAS) have identified genetic variants contributing to the risk of cardiovascular disease

\footnotetext{
This study was supported in part by grants-in-aid from the Program for Promotion of Fundamental Studies in National Institute of Biomedical Innovation of Japan (HR: 22-2-5), the Ministry of Education, Culture, Sports, Science, and Technology of Japan (KK: 22510211) and NOVARTIS Foundation for Gerontological Research (KK).

* Corresponding author at: Department of Geriatric Medicine and Nephrology, Osaka University Graduate School of Medicine, 2-2 Yamadaoka (B6), Suita, Osaka 565-0871, Japan. Tel.: +8166879 3852; fax: +8166879 3859 .

E-mail address: ohishi@geriat.med.osaka-u.ac.jp (M. Ohishi).
}

(CVD) at the chromosome 9p21 locus [1,2]. The CVD-associated regions are adjacent to the last exons of a long noncoding RNA (IncRNA), ANRIL (also known as CDKN2BAS). Two cyclindependent kinases inhibitors, CDKN2A and $C D K N 2 B$ (encoding $\mathrm{p} 15^{\mathrm{INK} 4 \mathrm{~B}}, \mathrm{p} 16^{\mathrm{INK} 4 \mathrm{~A}}$, and $\mathrm{p} 14^{\mathrm{ARF}}$ ) lie close to the association spot ( $\sim 100 \mathrm{~kb}$ from the associated SNPs). These kinase inhibitors are key players in cell proliferation and are appealing candidates to mediate these associations.

$C D K N 2 A / B$ are known to be repressed by Polycomb proteins during cell growth and then activated during senescence [3]. There is strong evidence to support the role of ANRIL in the regulation of 


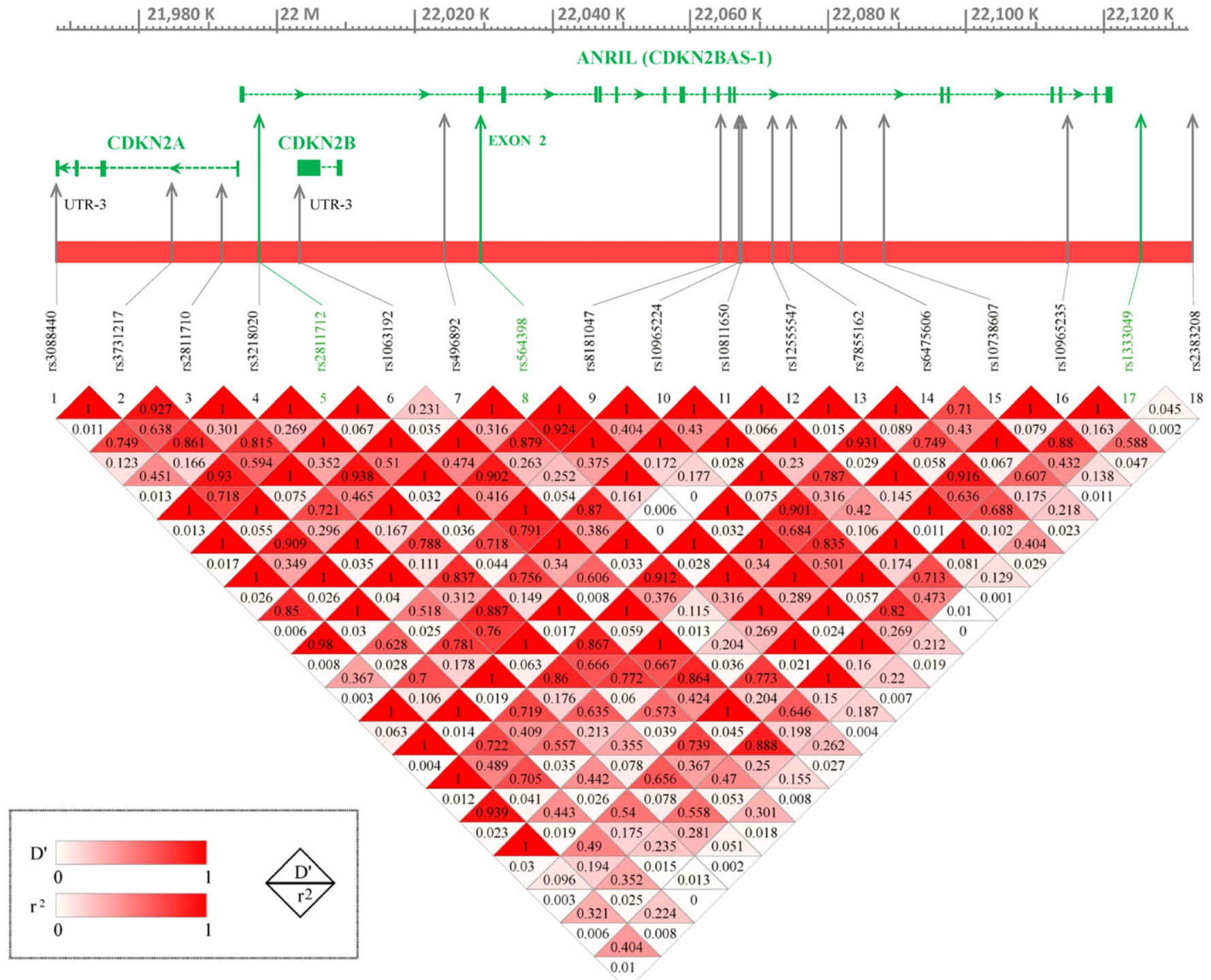

Fig. 1. Linkage disequilibrium (LD) and transcriptional map of the 9p21 locus.

the $C D K N 2 A / B$ locus through histone modification [4,5]. ANRIL has been proposed to regulate senescence at the CDKN2A locus, and it showed a senescence-dependant role in proliferation [4]. The transcript complexes with Chromobox 7 (CBX7 subunit of Polycomb repressive complex 1$)$ to silence $\mathrm{p} 16^{\mathrm{INK} 4 \mathrm{~A}}(C D K N 2 A)[4]$ and it is crucial in the recruitment of Polycomb repressive complex 2 (PRC2) to silence $\mathrm{p} 15^{\mathrm{INKB}}$ (CDKN2B) [5].

We investigated the association between single nucleotide polymorphisms (SNPs) in the region with expression of ANRIL as well as the genes encoded in this locus and explored the possible role of this non-coding RNA in $C D K N 2 A / B$ regulation in vascular smooth muscle cells (VSMCs), which are directly involved in the pathogenesis of atherosclerosis.

The chromosome 9p21 region is particularly abundant in disease-associated SNPs, some phenotypes associated with the locus are connected with vascular disease such as atherosclerotic stroke, CVD, myocardial infarction, and more indirectly intracranial aneurism and type-2 diabetes [1,2,6,7], but, surprisingly, also with other conditions of more distant etiologies like melanoma [8], cutaneous nevi [9], leukemia [10], periodontitis [11,12] and Alzheimer's disease [13,14], endometriosis [15] and more recently with glaucoma $[16,17]$. Here, we provide evidence of the influence of several SNPs in the region upon the expression of these transcripts, ultimately linking them with human atherosclerosis, and a role of ANRIL in regulating CDKN2A/B expression in aortic VSMC and proliferation.

\section{Methods}

\subsection{Study sample}

For the genetic association study between the SNPs: rs1333049, rs564398 and rs2811712 (see SNPs in green in Fig. 1), and CAD, patients with documented evidence of angiographically diagnosed coronary stenosis (including acute myocardial infarction, angina pectoris and, silent myocardial ischemia) (subjects characteristics in Supplementary Table 1 and Supplementary Methods).

For peripheral blood RNA expression analysis, we obtained whole blood samples from 57 volunteers (69-72 years old) from Asago City, Hyogo Prefecture, Japan (sample characteristics in Supplementary Table 2) who were participants in "SONIC" (Septuagenarians, Octogenarians, Nonagenarians Investigation with Centenarians) Study. Carotid ultrasonography using GE LOGIQ Book X-P was performed to measure the intima-media 

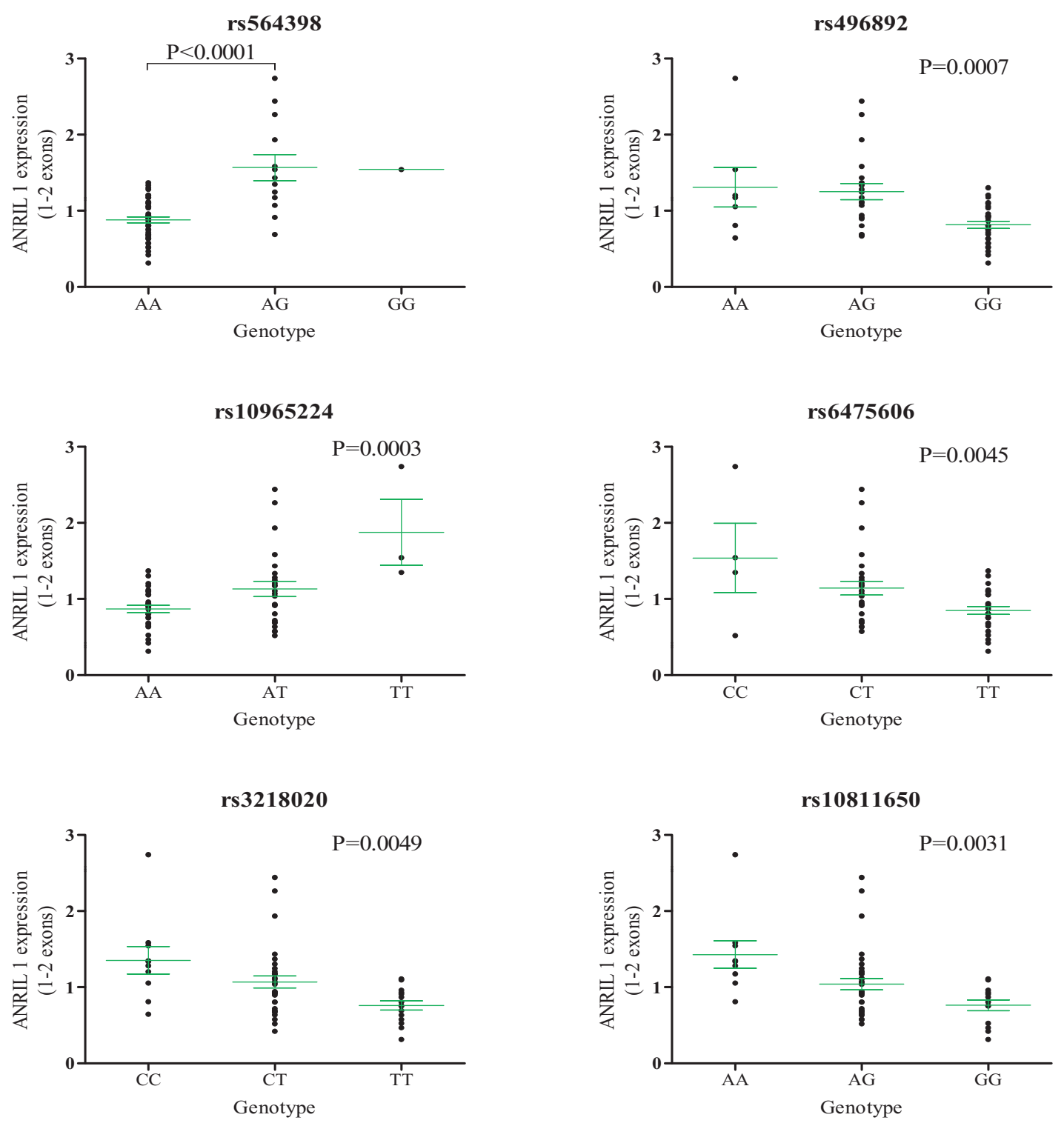

rs1063192
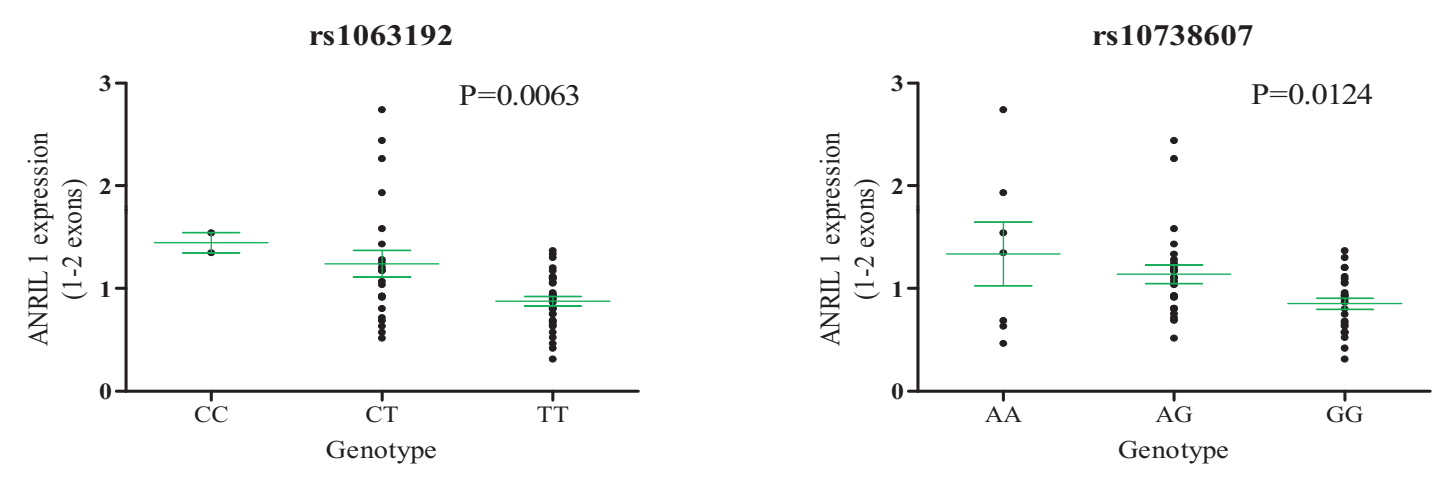

Fig. 2. ANRIL (exons 1-2) expression among genotypes in peripheral blood.

thickness (IMT) of the common carotid artery (CCA) for the evaluation of human atherosclerosis in 56 SONIC subjects (details in Supplementary Methods). We defined the criteria for CCA atherosclerosis as maximal-IMT $\geq 1.1 \mathrm{~mm}$. All participants gave written informed consent to participate in the genetic analyses and in all other procedures associated with the study. The ethics committee of Osaka University approved the study protocol.

\subsection{Genotyping}

All SNPs were genotyped with the standard TaqMan PCR method using commercial TaqMan Assays (rs numbers of genotyped SNPs are shown in Supplementary Table 3; see Supplementary Methods). We selected three SNPs (rs1333049, rs564398, and rs2811712) for genotyping of subjects with coronary artery disease. Rs1333049 is 
Table 1

SNPs associated with expression of 9p21 transcripts.

\begin{tabular}{|c|c|c|c|}
\hline SNP & Disease association (risk allele) & Lower expression allele ${ }^{a}$ & ANOVA $P$-value \\
\hline \multicolumn{4}{|l|}{ ANRIL (1-2 exons) expression } \\
\hline$\underline{\text { rs4966892 }}$ & Atherosclerotic stroke (G) [7] and periodontitis [12] & G & 0.0007 \\
\hline rs10738607 & & G & 0.0124 \\
\hline$\overline{\text { rs10811650 }}$ & & G & 0.0031 \\
\hline$\underline{\text { rs564398 }}$ & $\begin{array}{l}\text { Atherosclerotic stroke (A) [7], coronary artery } \\
\text { calcification [31] (A) and diabetes [19] (A) }\end{array}$ & A & $<0.0001$ \\
\hline rs3218020 & & $\mathrm{T}$ & 0.0049 \\
\hline rs6475606 & Coronary Heart Disease $(\mathrm{T})[32]$ & $\mathrm{T}$ & 0.0045 \\
\hline$\overline{\text { rs10965224 }}$ & & A & 0.0003 \\
\hline$\underline{\text { rs1063192 }}$ & $\begin{array}{l}\text { Myocardial infarction in Chinese }(\mathrm{T}) \text { [30], open angle } \\
\text { glaucoma (C) [17] }\end{array}$ & $\mathrm{T}$ & 0.0063 \\
\hline \multicolumn{4}{|l|}{ ANRIL (17-18 exons) expression } \\
\hline$\underline{\text { rs2811712 }}$ (AA vs. GG+AG) & Severe physical impairment in the elder (A) [20] & $\mathrm{GG}+\mathrm{AG}$ & 0.0426 \\
\hline rs10965235 (AC vs. CC) & Endometriosis (GWA) (C) [15] & AC & 0.0275 \\
\hline$\overline{\text { rs3731217 }}(\mathrm{GG}+\mathrm{GT}$ vs. TT) & Acute lymphoblastic leukemia [10] & $\mathrm{GG}+\mathrm{GT}$ & 0.0097 \\
\hline$\overline{\text { rs7855162 }}(\mathrm{CC}+\mathrm{CT}$ vs. TT $)$ & & $\mathrm{CC}+\mathrm{CT}$ & 0.0286 \\
\hline \multicolumn{4}{|l|}{ CDKN2A expression } \\
\hline rs10965235(AA+AG vs. GG) & Endometriosis (GWA) (C) [15] & $\mathrm{AA}+\mathrm{AG}$ & 0.0414 \\
\hline \multicolumn{4}{|l|}{ CDKN2B expression } \\
\hline rs2383208 (AA vs. AG+GG) & Diabetes in Japanese (A) [33] & $A G+G G$ & 0.0317 \\
\hline rs10738607 & & c & 0.0451 \\
\hline
\end{tabular}

a Allele associated with lower expression of 9p21 transcripts.

b Only one subject had AA genotype, see Supplementary Fig. 3.

c AG genotype was associated with increased expression of CDKN2B, see Supplementary Table 4.

the leading CAD-associated SNP [1]. Rs564398 is the SNP showing the strongest association with ANRIL expression in our analysis, as well as in a previous study [18], and has been associated with atherosclerotic stroke [7] and diabetes [19]. Rs2811712 was associated with frailty [20] and, due to its proximity with the CADassociated locus, we hypothesized a possible association with CAD.

For expression analysis, 18 tag SNPs were selected for genotyping $\left(r^{2}<0.8\right.$ and MAF $\left.>0.05\right)$ using Haploview software in the region of interest: $C D K N 2 A / B$ and ANRIL, and encompassing the CAD-associated region. After genotyping, SNP rs8181047 was in tight linkage disequilibrium $\left(r^{2}>0.9\right)$ with rs564398 in our sample, and it was excluded from further analysis (see Fig. 1). The tag SNPs and relevant associations are presented in Supplementary Table 3.

\subsection{Cell culture}

The culture media was Dulbecco's Modified Eagles Medium (DMEM) supplemented with $10 \%$ FBS and antibiotics $1 \%(v / v)$. For siRNA transfection, VSMC were incubated in DMEM supplemented with $10 \%$ FBS only, as recommended by the transfection agent manufacturer (see details in Supplementary Methods).

\subsection{ANRIL silencing}

Custom silencer select siRNA ${ }^{\circledR} S$ (Ambion) was designed to target ANRIL (NCBI Reference Sequence: NR_003529.3) in its exon 19 (see Fig. 4B and Supplementary Methods). Universal negative control siRNA \#1 (Ambion) was used as a control for all the experiments. Lipofectamine RNAimax was purchased from Invitrogen and used as a transfection reagent according to the manufacturer's instructions (details in supplementary Methods). The cells were incubated for $48 \mathrm{~h}$ before RNA extraction for RT-PCR evaluation and $72 \mathrm{~h}$ before the proliferation assay.

\subsection{RNA isolation and real time PCR}

See Supplementary Methods.

\subsection{Cell proliferation assay}

Aortic VSMC were cultured in 96-well plates and cell proliferation was measured using a cell proliferation colorimetric assay (CCK-8, Dojindo) according to the manufacturer's instructions. Absorbance was measured $72 \mathrm{~h}$ after treatment with siRNA using a microplate reader (Multi-Spectrophotometer, Viento, Dainippon) at a wavelength of $450 \mathrm{~nm}$.

\subsection{Immunostaining in mouse atherosclerotic plaque}

C57BL/6JJcl wild type and $\mathrm{APOE}^{-1-}$ mice (C57BL/6JJcl background) fed a normal diet, were used to obtain normal and atherosclerotic aortic sections at the age of 24 weeks (see Supplementary Methods).

\subsection{Statistical analysis}

Expression and genotype association results were analyzed using JMP software (version 4, SAS Institute Inc.) and Prism 5 for Windows (version 5.02, GraphPad Software Inc.). The results were analyzed using analysis of variance (ANOVA) and the $t$-test. $P$-values lower than 0.05 were considered significant.

\section{Results}

\subsection{Confirmation of 9p21 association in the Japanese sample}

We assessed the possible association of the three SNPs: rs1333049, rs564398, and rs2811712 with CAD. We confirmed the rs1333049 association with CAD in our sample (Controls CC:101; CG:224; GG:122; Cases CC:72; CG:116; GG:43; age adjusted $P$ value < 0.01 ) (Fig. 2A); however, we were not able to detect any significant association of CAD with either rs564398 or rs2811712 (see Supplementary Table 4).

\subsection{Expression analysis of $C D K N 2 A / B$ and ANRIL in blood}

The linkage disequilibrium (LD) map for the SNPs genotyped in this study and a transcriptional representation of the locus are 

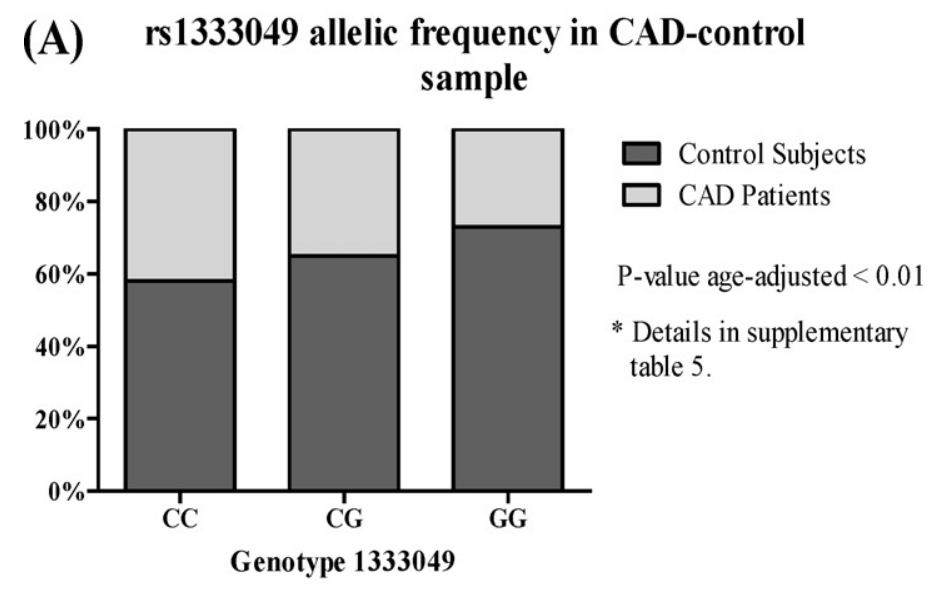

\section{(B) ANRIL expression and common carotid artery atherosclerosis}

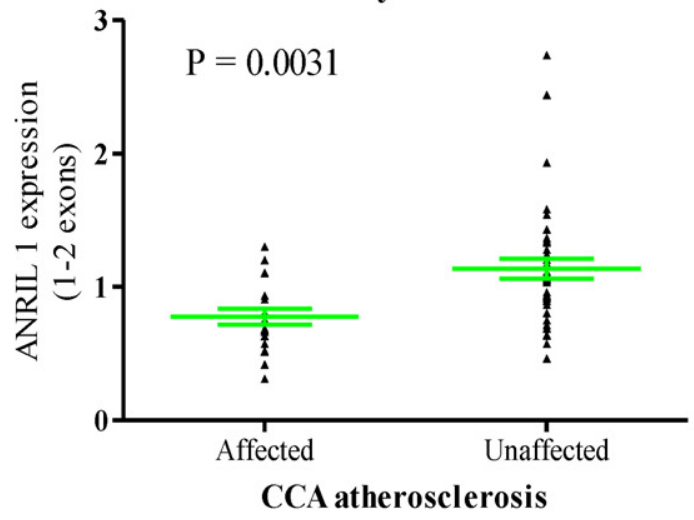

* CCA ultras onography information was available for 56 subjects

\section{(C) Immunostaining}

\section{Atherosclerotic Aorta Section in APOE KO Mice}

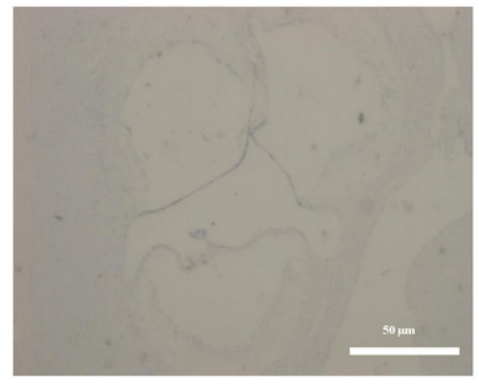

(-) control

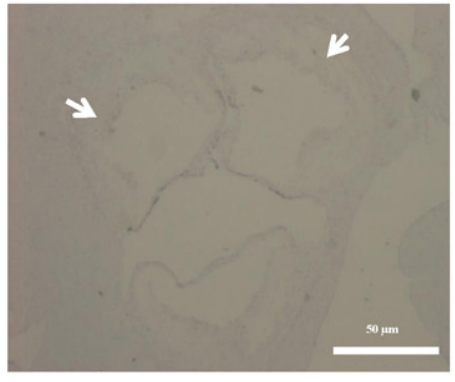

CDKN2A

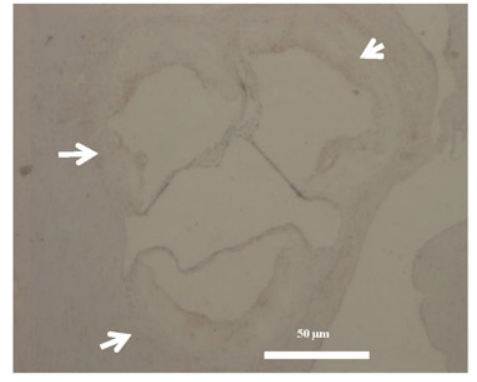

CDKN2B

\section{Aorta Section in Wild-Type Mice}

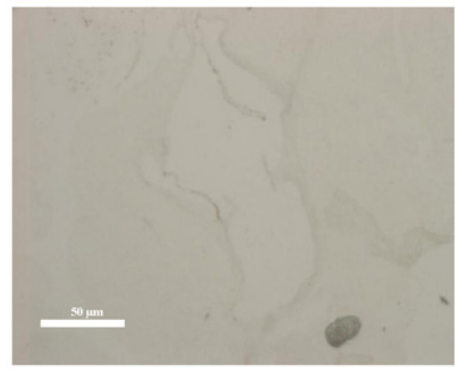

(-) control

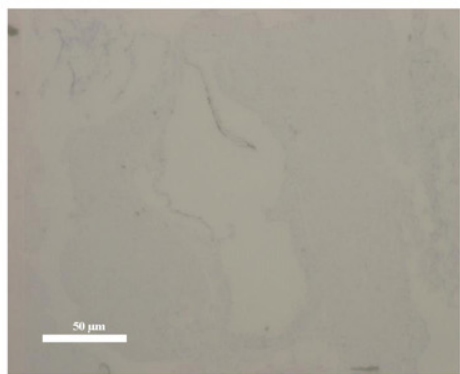

CDKN2A

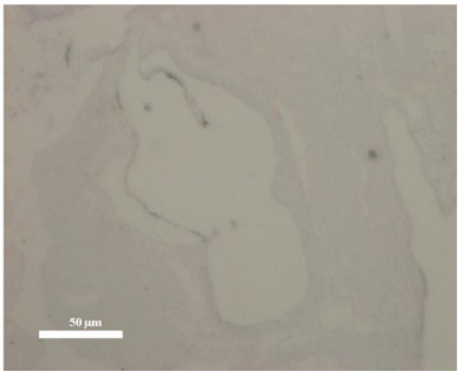

CDKN2B

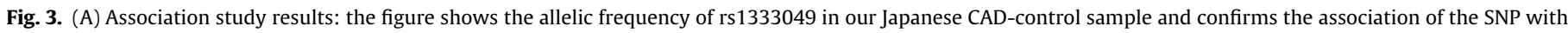

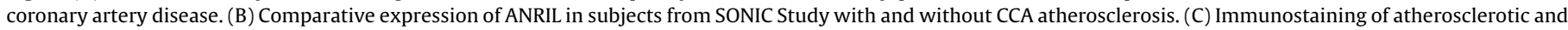
normal aortic sections with antibodies against CDKN2A, B and negative control.

presented in Fig. 1. Expression levels of $C D K N 2 A, C D K N 2 B$, and ANRIL (with the exception of ANRIL in exons 17-18 with CDKN2B) were positively correlated (correlation plots, $r$ and $P$-values shown in Supplementary Fig. 1), consistent with previous reports suggesting that the expression of these genes is co-regulated [18,21]. Common carotid artery atherosclerosis, a known predictor of ischemic stroke [22], was associated with a significantly lower expression of ANRIL (exons 1-2) ( $P$-value < 0.01 ) (Fig. 2B).

Several SNPs showed an effect in the expression of the 9p21 locus transcripts as shown in Table 1 and Supplementary Figs. 3-5; and the most significant effects detected in this study affected ANRIL (exons 1-2) expression as shown in Fig. 3. Remarkably most of the risk alleles for atherosclerosis-related phenotypes (all except rs1333049, which did not reach significance) were associated with a lower expression of the transcript, at least when evaluating the exons 1-2 (see Fig. 3). The leading CAD-associated SNP, rs1333049 was not significantly associated with the expression of either of the transcripts but when grouped risk allele versus others, the $P$-value was 0.0653 (Supplementary Fig. 5), and again, the risk allele seemed to be associated with a lower expression of the first exons of ANRIL. 
(A) ANRIL knock down efficiency (left), CDKN2A (center) and CDKN2B (right) expression after ANRIL Knock down
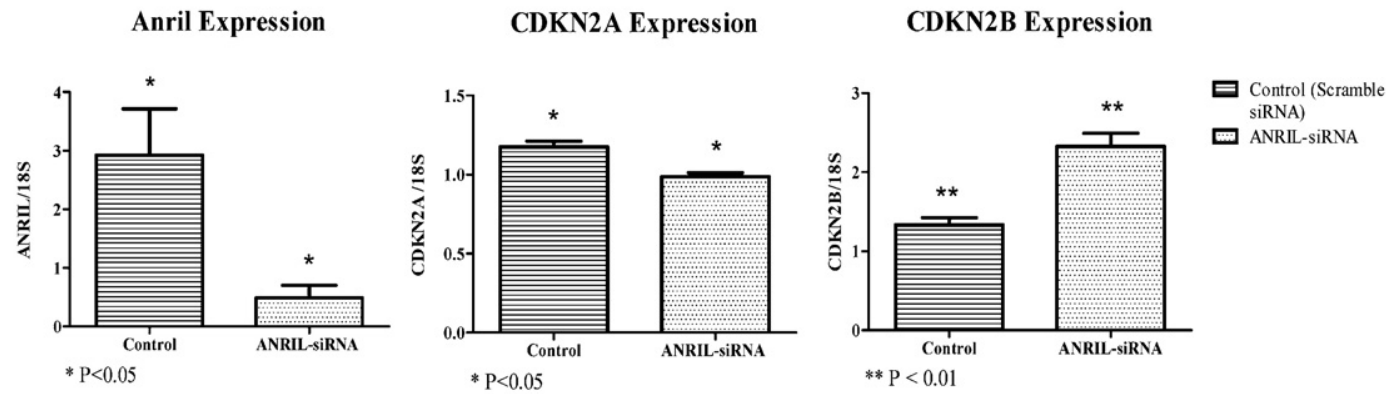

(B) RT-PCR primers and ANRIL-siRNA target
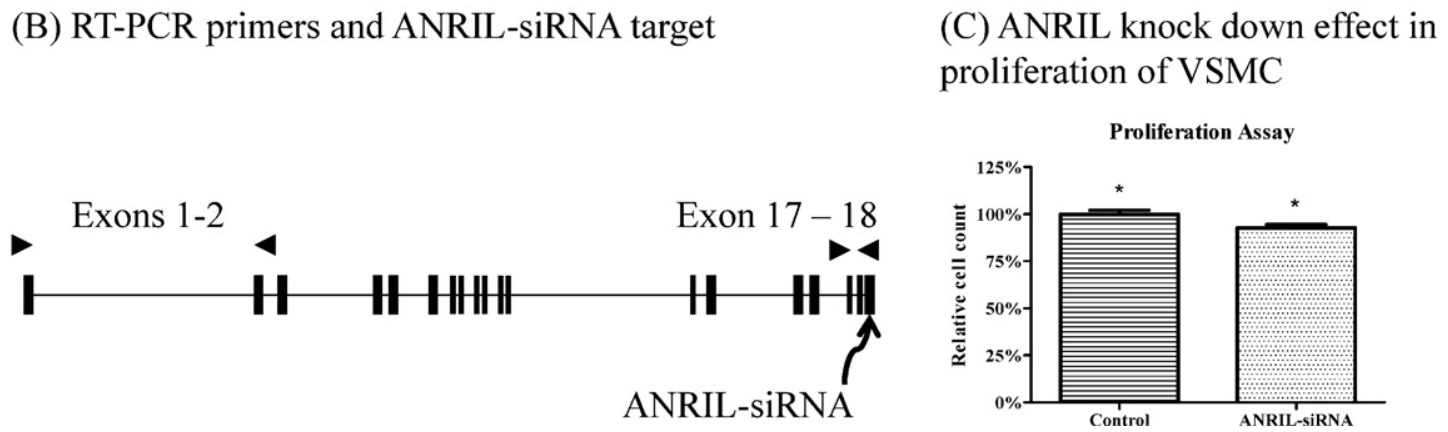

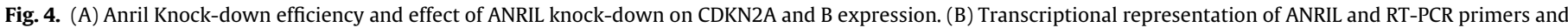
siRNA target location. (C) Cell proliferation results: relative cell counts of VSMCs following ANRIL-siRNA treatment.

\subsection{Evidence of ANRIL-mediated regulation of $C D K N 2 A / B$ expression}

We performed siRNA knock-down of ANRIL in VSMC, as described in Section 1, and examined the expression of $C D K N 2 A / B$. The knock-down efficiency was confirmed by RT-PCR and ANRIL expression is presented in Fig. 4A and Supplementary Fig. 6. We noted a marked increase in the expression of $C D K N 2 B$ and, interestingly, a slight but significant decrease in CDKN2A expression as shown in Fig. 4A.

\subsection{ANRIL affects proliferation in VSMC}

We measured proliferation in VSMC $72 \mathrm{~h}$ after siRNA treatment. ANRIL knock-down caused a reduced viable cell count in VSMC consistent with the over-expression of CDKN2B. The relative cell counts are shown in Fig. 4C.

\section{5. $C D K N 2 A / B$ expressed in mouse atherosclerotic plaque}

Since our results linked the expression of ANRIL with risk alleles at the atherosclerosis-associated 9p21 locus, and also suggested that ANRIL is involved in the regulation of $C D K N 2 A / B$, we considered relevant to investigate the expression of these genes in atherosclerotic plaque in vivo. We detected the presence of both $C D K N 2 A$ and $C D K N 2 B$ in mouse aortic plaque on immunostaining, which supports our hypothesis that $C D K N 2 A / B$ genes at least partially mediate the genetic susceptibility to atherosclerosis found at the locus 9p21 (Fig. 3C).

\section{Discussion}

Since the GWAs have revealed a CAD association at the 9p21 locus, researchers have hypothesized a potential regulatory role of the associated SNPs in the expression of ANRIL, CDKN2A and $C D K N 2 B$. However, publications demonstrating a correlation between the risk genotypes and the expression of the transcripts in the locus have reported contradictory results [18,23,24], highlighting the need to replicate such studies to clarify true associations. Probably, this lack of consistency is due to the splicing complexity of ANRIL and characteristics specific to the sample (ethnicity, age, health status, etc.). Since CDKN2A is a known marker of aging [25] and increases in expression with age in several tissues of rodents and humans [26] and ANRIL has been connected with cell senescence [4], we believe the very narrow age range (69-72 years old) of our subjects maximizes the detection of possible genotype effects. Here, we showed that several SNPs in the region affect the expression of the locus, particularly in the case of previously disease-associated SNPs. CDKN2A/B were prime candidates in the association of these SNPs, however, most of the significant associations found in this report pointed at ANRIL rather than $C D K N 2 A / B$. Despite the limitation of this study regarding the number of subjects, we were able to reproduce previously reported associations, as in the case of rs564398 and rs496892 and their effect on ANRIL expression [18]. Remarkably, the vascular disease related risk alleles seem to be consistently associated with the under-expression of the first exons of ANRIL at least in the peripheral blood. The CCA atherosclerosis phenotype was also correlated with reduced expression of ANRIL, with both results pointing to a possible protective role of ANRIL in the progression of atherosclerosis.

ANRIL is antisense to CDKN2B (also known as p15), a situation that typically implies an inhibitory effect in the sense sequence expression. Here, we established a correlation between the expression of ANRIL and $C D K N 2 A / B$ in human aortic VSMC. The effect of ANRIL in CDKN2B expression identified in this report is consistent with the previously reported role of ANRIL in the repression of the locus $C D K N 2 A / B$ [4]. Accordingly, in the situation of the 
absence or reduction of ANRIL (by knock-down), the repression of the locus would be impaired and $C D K N 2 B$ would be up-regulated. On the other hand, the PR complex hypothesis does not explain the reduction of CDKN2A following ANRIL silencing which seems to be operated by another mechanism. Even though the role of ANRIL in the repression of the locus CDKN2A/B through Polycomb proteins has been highlighted in recent publications, the specific role of each splicing variant of ANRIL as well as the totality of possible functions of the transcripts are still poorly understood. A clue to the wide spectrum of ANRIL functions is provided by Sato et al. [27], where a subset of genes involved in the regulation of the nucleus and chromatin architecture were up-regulated after the over-expression of a novel splicing variant of ANRIL. We believe that the repression of CDKN2A presented in this report is due to mechanisms that may be specific to VSMCs or to the splicing variants targeted in the experiment. Additionally, the reduction in proliferation caused by ANRIL knock down is consistent with the increased expression of anti-proliferative gene CDKN2B. Although, the deletion of the CAD risk region in a mouse model leaded to an exacerbated proliferation in SMC [28], which seems inconsistent with the results presented here, another report showed that inhibition of ANRIL causes reduced proliferation in human fibroblasts [4]. ANRIL does not have a homologous transcript described in mice, and ANRIL functions could be specific to humans.

These findings emphasize the importance of ANRIL in cell proliferation and regulation of the locus $C D K N 2 A / B$ in a cell line directly involved in the pathogenesis of atherosclerosis. Overall, our results suggest that several SNPs in 9p21 locus affect the expression of ANRIL, which is further in control of the regulation of $C D K N 2 A / B$ and cell growth. Cell proliferation mediates the progression of atherosclerosis and is also directly or indirectly involved in the pathogenesis of diseases associated with this locus.

In our study, expression of exons 17-18 was strongly reduced confirming ANRIL-siRNA efficiency, however, expression of exons 1-2 did not show significant reduction (Supplementary Fig. 6) suggesting the existence of novel splicing variants at least in VSMC (possibly comprising the last exons but lacking the first ones). Further characterization of all ANRIL splicing variants and their functions is needed for a better understanding of their role in cell physiology, atherosclerosis, and ultimately human health. However, our results point at ANRIL as a potential major player in human vascular disease.

\section{Acknowledgements}

We would like to express our gratitude to Ms. Yuki Imaizumi and Ms. Takako Baba for their continuous support in this investigation.

\section{Appendix A. Supplementary data}

Supplementary data associated with this article can be found, in the online version, at doi:10.1016/j.atherosclerosis.2011.11.017.

\section{References}

[1] The Wellcome Trust Case Control Consortium. Genome-wide association study of 14,000 cases of seven common diseases and 3000 shared controls. Nature 2007;447:661-78.

[2] Broadbent HM, Peden JF, Lorkowski S, et al. Susceptibility to coronary artery disease and diabetes is encoded by distinct, tightly linked, SNPs in the ANRIL locus on chromosome 9p. Hum Mol Genet 2008;17:806-14.

[3] Gil1 J, Peters G. Regulation of the INK4b-ARF-INK4a tumour suppressor locus: all for one or one for all. Nat Rev Mol Cell Biol 2006;7:667-77.

[4] Yap KL, Li S, Muñoz-Cabello AM, et al. Molecular Interplay of the noncoding RNA ANRIL and methylated histone H3 lysine 27 by Polycomb CBX7 in transcriptional silencing of INK4a. Mol Cell 2010;38:662-74.
[5] Kotake Y, Nakagawa T, Kitagawa K, et al. Long non-coding RNA ANRIL is required for the PRC2 recruitment to and silencing of p15INK4B tumor suppressor gene. Oncogene 2011;30:1956-62.

[6] Helgadottir A, Thorleifsson G, Magnusson KP, et al. The same sequence variant on 9p21 associates with myocardial infarction, abdominal aortic aneurysm and intracranial aneurysm. Nat Genet 2008;40:217-24.

[7] Gschwendtner A, Bevan S, Cole JW, et al. International stroke genetics consortium, sequence variants on chromosome 9p21. 3 confer risk for atherosclerotic stroke. Ann Neurol 2009;65:531-9.

[8] Yang XR, Liang X, Pfeiffer RM, et al. Associations of 9p21 variants with cutaneous malignant melanoma, nevi, and pigmentation phenotypes in melanoma-prone families with and without CDKN2A mutations. Familial Cancer 2010;9:625-33.

[9] Falchi M, Bataille V, Hayward NK, et al. Genome-wide association study identifies variants at 9p21 and 22q13 associated with development of cutaneous nevi. Nat Genet 2009;41:915-9.

[10] Sherborne AL, Hosking FJ, Prasad RB, et al. Variation in CDKN2A at 9p21.3 influences childhood acute lymphoblastic leukemia risk. Nat Genet 2010;42:492-4

[11] Schaefer AS, Richter GM, Groessner-Schreiber B, et al. Identification of a shared genetic susceptibility locus for coronary heart disease and periodontitis. PLoS Genet 2009;5:e1000378.

[12] Ernst FD, Uhr K, Teumer A, et al. Replication of the association of chromosomal region 9p21.3 with generalized aggressive periodontitis (gAgP) using an independent case-control cohort. BMC Med Genet 2010;11:119.

[13] Yu JT, Yu Y, Zhang W, et al. Single nucleotide polymorphism rs1333049 on chromosome 9p21.3 is associated with Alzheimer's disease in Han Chinese. Clin Chim Acta 2010;411:1204-7.

[14] Emanuele E, Lista S, Ghidoni R, et al. Chromosome 9p21.3 genotype is associated with vascular dementia and Alzheimer's disease. Neurobiol Aging 2011;32:1231-5.

[15] Uno S, Zembutsu H, Hirasawa A, et al. A genome-wide association study identifies genetic variants in the CDKN2BAS locus associated with endometriosis in Japanese. A genome-wide association study identifies genetic variants in the CDKN2BAS locus associated with endometriosis in Japanese. Nat Genet 2010;42:707-10.

[16] Burdon KP, Macgregor S, Hewitt AW, et al. Genome-wide association study identifies susceptibility loci for open angle glaucoma at TMCO1 and CDKN2BAS1. Nat Genet 2011;43:574-8.

[17] Ramdas WD, van Koolwijk LM, Lemij HG, et al. Common genetic variants associated with open-angle glaucoma. Hum Mol Genet 2011;20:2464-71.

[18] Cunnington MS, Koref MS, Mayosi BM, Burn J, Keavney B. Chromosome 9p21 SNPs associated with multiple disease phenotypes correlate with ANRIL expression. PLoS Genet 2010;6:e1000899.

[19] Cugino D, Gianfagna F, Santimone I, et al. Type 2 diabetes and polymorphisms on chromosome 9p21: a meta-analysis. Nutr Metab Cardiovasc Dis NMCD 2011;(April), doi:10.1016/j.numecd.2010.11.010 [Epub ahead of print].

[20] Melzer D, Frayling TM, Murray A, et al. A common variant of the p16INK4a genetic region is associated with physical function in older people. Mech Ageing Dev 2007; 128:370-7.

[21] Pasmant E, Laurendeau I, Héron D, Vidaud M, Vidaud D, Bièche I. Characterization of a germ-line deletion. Including the entire INK4/ARF locus, in a melanoma-neural system tumor family: identification of ANRIL, an antisense noncoding RNA whose expression coclusters with ARF. Cancer Res 2007;67:3963.

[22] Alexandrov AV. Cerebrovascular ultrasound in stroke prevention and treatment. Blackwell Publishing; 2004. p. 161-9 [chapter 9].

[23] Jarinova O, Stewart AFR, Roberts R, et al. Functional analysis of the chromosome 9p21.3 coronary artery disease risk locus. Arterioscler Thromb Vasc Biol 2009, doi:10.1161/atvbaha.109.189522.

[24] Holdt LM, Beutner F, Scholz M, et al. ANRIL expression is associated with atherosclerosis risk at chromosome 9p21. Arterioscler Thromb Vasc Biol 2010, doi:10.1161/atvbaha.109.196832.

[25] Liu Y, Sanoff HK, Cho H, et al. Expression of p16INK4a in peripheral blood T-cells is a biomarker of human aging. Aging Cell 2009;4:439-48.

[26] Kim WY, Sharpless NE. The regulation of INK4/ARF in cancer and aging. Cell 2006;127:265-75.

[27] Sato K, Nakagawa H, Tajima A, Yoshida K, Inoue I. ANRIL is implicated in the regulation of nucleus and potential transcriptional target of E2F1. Oncol Rep 2010;24:701-7.

[28] Visel A, Zhu Y, May D, et al. Targeted deletion of the 9p21 non-coding coronary artery disease risk interval in mice. Nature 2010;464:409-12.

[29] Chen J, Li D, Wei C, et al. Aurora-A and p16 polymorphisms contribute to an earlier age at diagnosis of pancreatic cancer in Caucasians. Clin Cancer Res 2007; 13:3100-4.

[30] Yang XC, Zhang Q, Chen ML, et al. MTAP and CDKN2B genes are associated with myocardial infarction in Chinese Hans. Clin Biochem 2009;42:1071-5.

[31] Pechlivanis S, Scherag A, Mühleisen TW, et al. Coronary artery calcification and its relationship to validated genetic variants for diabetes mellitus assessed in the Heinz Nixdorf recall cohort. Arterioscler Thromb Vasc Biol 2010;30:1867-72.

[32] McPherson R, Pertsemlidis A, Kavaslar N, et al. A common allele on chromosome 9 associated with coronary heart disease. Science 2007;316:1488-91.

[33] Takeuchi F, Serizawa M, Yamamoto K, et al. Confirmation of multiple risk Loci and genetic impacts by a genome-wide association study of type 2 diabetes in the Japanese population. Diabetes 2009;58:1690-9. 